3-1983

\title{
Quantum Theory of Slow Atomic Collisions
}

John B. Delos

William \& Mary, jbdelos@wm.edu

Follow this and additional works at: https://scholarworks.wm.edu/aspubs

Part of the Physics Commons

\section{Recommended Citation}

Delos, John B., Quantum Theory of Slow Atomic Collisions (1983). International Journal of Quantum Chemistry, 24(S17), 37-48.

https://doi.org/10.1002/qua.560240806

This Article is brought to you for free and open access by the Arts and Sciences at W\&M ScholarWorks. It has been accepted for inclusion in Arts \& Sciences Articles by an authorized administrator of W\&M ScholarWorks. For more information, please contact scholarworks@wm.edu. 


\title{
Quantum Theory of Slow Atomic Collisions
}

\author{
J. B. DELOS
}

Physics Department, The College of William and Mary, Williamsburg, Virginia 23185, USA

\begin{abstract}
Quantum-mechanical and semiclassical theories of slow atomic collisions are reviewed, with attention to electron-translation factors and their effects.
\end{abstract}

\section{Introduction}

We consider here some of the most general aspects of the theory of ion-atom or atom-atom collisions, reviewing some pioneering steps taken by Bates and his collaborators, and discussing more recent contributions, especially focusing on ideas that were suggested by Russek, Mittleman, Green, Thorson, and others [1]. We restrict ourselves mainly to slow collisions, in which the nuclear velocity is much less than the electron velocity. For concreteness, we will consider collisions between protons and hydrogen atoms

$$
\mathrm{H}^{+}+\mathrm{H}(1 s) \rightarrow \text { products, }
$$

but most of our discussion applies to all atomic collisions.

There are two ways of giving a theoretical description of such processes. In a fully quantum-mechanical description, the wave function $\Psi(\mathbf{r}, \mathbf{R})$ depends on nuclear coordinates $(\mathbf{R})$ and electron coordinates $(\mathbf{r})$, and it satisfies the full Schrödinger equation

$$
\begin{gathered}
H \Psi(\mathbf{R}, \mathbf{r})=E \Psi(\mathbf{R}, \mathbf{r}), \\
H=-\left(\hbar^{2} / 2 \mu\right) \nabla_{R}^{2}+h(\mathbf{r} ; \mathbf{R}), \\
h(\mathbf{r} ; \mathbf{R})=-\left(\hbar^{2} / 2 m\right) \nabla_{r}^{2}+V(r ; R) .
\end{gathered}
$$

This is in principle the most complete description, but for many collision systems it is unnecessarily complicated, and a classical trajectory approximation is preferable. In this approach, it is assumed that the nuclei move on a classical path, and that the electron wave function $Y(r, t)$ satisfies a time-dependent Schrödinger equation

$$
h(\mathbf{r}, \mathbf{R}(t)) Y(\mathbf{r}, t)=i \hbar \partial / \partial t \partial Y(\mathbf{r}, t)
$$




\section{Classical Trajectory Theory}

Usually Eqs. (2) or (4) are solved by an expansion (the "close-coupling" method) and we will consider the classical trajectory description first. Let $\left\{\phi_{n}(\mathbf{r} ; \mathbf{R})\right\}$ be some carefully chosen set of electronic states, suitable to the system at hand, and expand [2]

$$
Y(\mathbf{r}, t)=\sum_{n} b_{n}(t) \phi_{n}(\mathbf{r} ; \mathbf{R}(t)) .
$$

The coefficients $\left\{b_{n}(t)\right\}$ then satisfy coupled differential equations, which can be written in matrix form

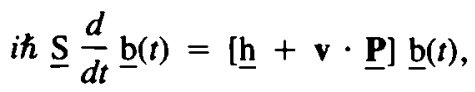

with

$$
\begin{aligned}
& S_{m n}=\left\langle\phi_{m} \mid \phi_{n}\right\rangle, \\
& h_{m n}=\left\langle\phi_{m}|h| \phi_{n}\right\rangle, \\
& \mathbf{P}_{m n}=-i \hbar\left\langle\phi_{m} \mid \nabla_{R} \phi_{n}\right\rangle,
\end{aligned}
$$

$\underline{S}$ is the overlap, $\underline{h}$ is the Hamiltonian matrix, and $\underline{\mathbf{P}}$ represents the total rate of change of the basis functions with internuclear distance.

The above approach seems to be simple, general, and rigorous. Let us see what happens when it is applied to the proton-hydrogen system. In an early paper, Jepsen and Hirschfelder [3] took the $\phi_{n}$ 's to be Born-Oppenheimer states (eigenfunctions of $h$ ) and they computed (among other things) the coupling between $1 s \sigma_{g}$ and $2 s \sigma_{g}$ states. They found that the $P$-matrix element had a curious property: it did not go to zero as $R \rightarrow \infty$. If this were not corrected in some way, it would imply that the cross section for $1 s \rightarrow 2 s$ excitation would be infinite, a quite implausible result.

Bates and McCarroll [4] were the first to propose a resolution to this problem. To understand their approach, we have to consider expansions in atomic basis functions, such as might be useful for describing fast collisions. If initially the electron is in the ground state on proton $A$, then the wave function for this initial state is

$$
\Psi_{i}=\phi_{1 s}\left(\mathbf{r}-\mathbf{R}_{A}(t)\right) \exp \left[i m\left(v_{A} \cdot \mathbf{r}-\frac{1}{2} v_{A}{ }^{2} t\right) / \hbar\right],
$$

where $\mathbf{v}_{A}$ is the velocity of proton $A$. A corresponding final state with the electron on $B$ is

$$
\Psi_{f}=\phi_{1 s}\left(\mathbf{r}-\mathbf{R}_{B}(t)\right) \exp \left[i m\left(\mathbf{v}_{B} \cdot \mathbf{r}-\frac{1}{2} v_{B}^{2} t\right) / \hbar\right]
$$


The exponential factor is the "electron-translation-factor" (ETF), which describes the momentum and kinetic energy of the electron as it is carried with nucleus $A$ or $B$.

The presence of ETFS in the initial and final states suggests that we should include them explicitly in our expansion, taking

$$
\begin{aligned}
Y(\mathbf{r}, t)= & \sum_{n} b_{n}^{A}(t) F_{n}^{A}(\mathbf{r}, t) \phi_{n}^{A}\left(\mathbf{r}-\mathbf{R}_{A}(t)\right) \\
& +\sum_{n} b_{n}^{B}(t) F_{n}^{B}(\mathbf{r}, t) \phi_{n}^{B}\left(\mathbf{r}-\mathbf{R}_{B}(t)\right),
\end{aligned}
$$

where $F_{n}^{A . B}$ are the ETFs given in Eq. (8). Using Eq. (9) instead of Eq. (5), we arrive at a modified set of coupled equations for the new coefficients $\left\{b_{n}(t)\right\}$ :

$$
\begin{aligned}
i \hbar \underline{\mathrm{S}}(v) \frac{d}{d t} \underline{\mathrm{b}}(t)=\left[\underline{\mathrm{h}}(v)+\mathrm{v} \cdot \Pi^{\mathrm{o}}(v)\right] \underline{\mathrm{b}}(t), \\
S_{m n}(v)=\left\langle\phi_{m} \mid\left(F_{m}{ }^{*} F_{n}\right) \phi_{n}\right\rangle, \\
h_{m n}(v)=\left\langle\phi_{m} \mid\left(F_{m}{ }^{*} F_{n}\right) h \phi_{n}\right\rangle, \\
\mathbf{v} \cdot \Pi_{m n}^{0}(v)=\left\langle\phi_{m} \mid\left(F_{m}{ }^{*} F_{n}\right)(-i \hbar)\left(\frac{\partial}{\partial t}+\mathrm{v}_{n} \cdot \nabla_{r}\right) \phi_{n}\right\rangle .
\end{aligned}
$$

The factor $F_{m}^{*} F_{n}$ is called the momentum transfer factor. If the basis states $\phi_{m}, \phi_{n}$ are on the same nucleus, then $F_{m}^{*} F_{n}=1$, while if they are on different nuclei, $F_{m}{ }^{*} F_{n}=\exp ( \pm i m \mathbf{v} \cdot \mathbf{r})$ times a less important phase. In fast collisions, the momentum transfer factor is very important: it is responsible for the steep decline in charge exchange cross sections at high velocities. An illustration was given by McCarroll [5] and it is shown in Figure 1. One sees that at low velocities,

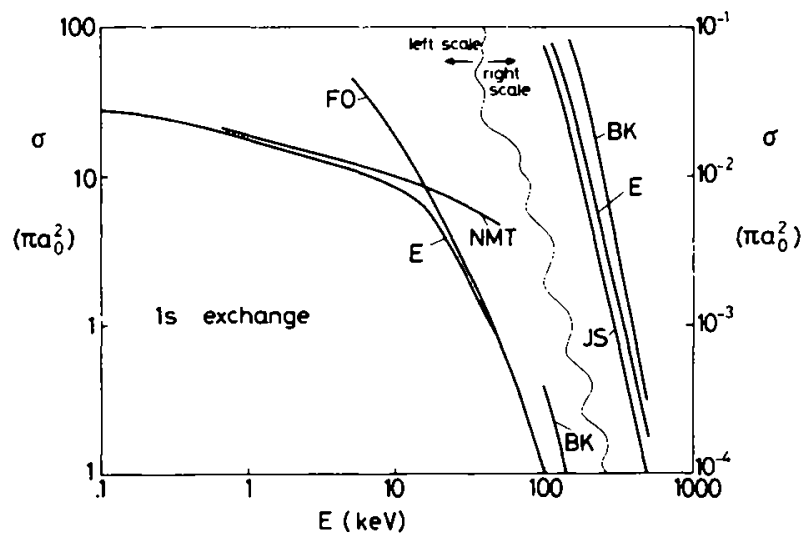

Figure 1. Total cross section for $\mathrm{H}^{+}+\mathrm{H}(1 s) \rightarrow \mathrm{H}(1 s)+\mathrm{H}^{+}$vs. energy. $\mathrm{E}$ : exact "one state" $\left(1 s_{A}, 1 s_{B}\right)$ result; NMT: neglecting momentum transfer factors, $\left(F_{A}^{*} F_{B}=1\right)$; BK: Brinkman and Kramers; Js: Jackson and Schiff; FO: type of "first order." 
the momentum transfer factor has no effect in this case, and for this reason ETFS were left out of collision calculations for many years.

The last part of Eq. (11c) is more subtle. The quantity $\left(\partial / \partial t+\mathbf{v}_{n} \cdot \nabla_{r}\right)\left|\phi_{n}\right\rangle$ represents the rate of change of $\phi_{n}$ as seen in a frame that is moving with the nucleus to which $\phi_{n}$ is attached. It is this that eliminates the infinite-range couplings that would otherwise appear.

We therefore see that for a proper theory, ETFs must be introduced even for slow collisions. However, it was not obvious how to incorporate them into an expansion involving molecular states. Bates and McCarroll suggested that if the molecular state goes asymptotically into an $A$ (or $B$ ) atomic state, then with it we can introduce an $A$-type (or $B$-type) translation factor, as in Eq. (8). This approach remedies the worst defects of the unmodified expansion (5), but a better idea was suggested a couple of years later by Schneiderman and Russek [6], then further developed and exploited by Thorson and co-workers $[7,8]$.

Their method involves associating with each molecular state a switching function $f_{n}(\mathbf{r} ; \mathbf{R})$ which varies smoothly between -1 and +1 ; then a local electron transport velocity is defined as

$$
\begin{aligned}
\mathbf{W}_{n}(\mathbf{r} ; \mathbf{R}) & =\frac{1}{2}\left[f_{n}(\mathbf{r} ; \mathbf{R})+\lambda\right] \mathbf{v}, \\
\lambda & =\left(M_{A}-M_{B}\right) /\left(M_{A}+M_{B}\right) .
\end{aligned}
$$

Those parts of the basis function that are near nucleus $A$ (or $B$ ) are assumed to be moving with the velocity of $A$ (or $B$ ), and the parts that are between the nuclei are moving slowly, or not at all. A possible form for the ETF is

$$
F_{n}=\exp \left[\operatorname{im} \mathbf{v} \cdot \mathbf{s}_{n}(\mathbf{r} ; \mathbf{R}) / \hbar\right],
$$

with

$$
s_{n}(\mathbf{r} ; \mathbf{R})=\frac{1}{2}\left[f_{n}(\mathbf{r} ; \mathbf{R})+\lambda\right] \mathbf{r}_{g}-\frac{1}{8}\left(1-\lambda^{2}\right) \mathbf{R},
$$

where $\mathbf{r}_{g}$ is the electron coordinate relative to the geometric center of the nuclei. When the ETF-modified molecular expansion

$$
Y(\mathbf{r}, t)=\sum_{n} b_{n}(t) F_{n}(\mathbf{r} ; \mathbf{R}(t)) \phi_{n}(\mathbf{r} ; \mathbf{R}(t))
$$

is put into the Schrödinger equation, a set of equations quite similar to Eqs. (10) and (11) is obtained, except that the derivatives of switching functions also appear. Those equations can be simplified by expanding the matrix elements in powers of velocity, neglecting terms of order $v^{2}$ and higher, as well as terms proportional to $d v / d t$. The result is a set of coupled equations that should be quite generally applicable to slow collisions

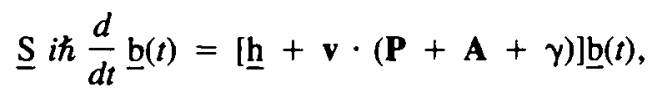

$\underline{S}, \underline{h}$, and $\underline{P}$ are the same matrices that appeared in Eq. (7), and 


$$
\begin{aligned}
\mathbf{A}_{k n} & =(i m / \hbar)\left\langle\phi_{k}\left|\left[h, \mathbf{s}_{n}\right]\right| \phi_{n}\right\rangle, \\
\boldsymbol{Y} & =\underline{\boldsymbol{\eta}}-\underline{\boldsymbol{\sigma}} \underline{\mathrm{S}}^{-1} \underline{\mathbf{h}}, \\
\boldsymbol{\eta}_{k n} & =(i m / \hbar)\left\langle\phi_{k}\left|\left(\mathbf{s}_{n}-\mathbf{s}_{k}\right) h\right| \phi_{n}\right\rangle, \\
\boldsymbol{\sigma}_{k n} & =(i m / \hbar)\left\langle\phi_{k}\left|\mathbf{s}_{n}-\mathbf{s}_{k}\right| \phi_{n}\right\rangle .
\end{aligned}
$$

These quantities have the following physical interpretation. As stated earlier, the matrix $\underline{P}$ represents the total rate of change of basis functions with internuclear separation, and this change arises from several sources. As the nuclei move, the basis functions must follow them, but also they may undergo polarization, distortion, and change of character. All of the latter can cause "real" nonadiabatic transitions, but the simple displacement of basis functions with moving nuclei does not cause transitions. The part of $\underline{\mathbf{P}}$ that only represents displacement may therefore be regarded as a "fictitious" coupling, and in fact it is cancelled by the matrix $\underline{\mathbf{A}}$. The quantity $\underline{\mathbf{P}}+\underline{\mathbf{A}}$ therefore corresponds to the adjusted time derivative that appears in $\mathrm{Eq}$. (11c): it represents the rate of changes other than displacement, and we refer to it as the "corrected nonadiabatic coupling."

The other matrix $\chi$ represents the low-velocity limit of the momentum transfer factors

$$
F_{k}^{*} F_{n}=1+(\mathrm{i} m / \hbar)\left(\mathrm{s}_{n}-\mathrm{s}_{k}\right) .
$$

Often this matrix may be negligible, and as of this writing it has not yet been calculated for any system.

\section{Quantum-Mechanical Theory}

We now turn to the fully quantum-mechanical description of atomic collisions, and we consider how we can calculate $\Psi$ in Eq. (2). Noting that the Born-Oppenheimer approximation

$$
\Psi(\mathbf{R}, \mathbf{r}) \simeq \chi(\mathbf{R}) \phi(\mathbf{r} ; \mathbf{R})
$$

has long been the basis of molecular theory, we may consider a simple generalization of it

$$
\Psi(\mathbf{R}, \mathbf{t})=\sum_{n} \chi_{n}(\mathbf{R}) \phi_{n}(\mathbf{r} ; \mathbf{R}) .
$$

This expansion leads to coupled equations for the functions $\chi_{n}(\mathbf{R})$ of the form

$$
\left\{(2 \mu)^{-1}\left[\underline{S}\left(-i \hbar \nabla_{R}\right)^{2}+2 \underline{P} \cdot-i \hbar \nabla_{R}+\underline{\mathrm{B}}^{0}\right]+\underline{\mathrm{h}}-E\right\}_{\chi}(R)=0,
$$

where

$$
B_{k n}^{0}=-\hbar^{2}\left\langle\phi_{k} \mid \nabla_{R}^{2} \phi_{n}\right\rangle
$$

and the other matrices are defined in Eq. (7). 
For many years, these equations were regarded as the foundation of the theory of slow collisions, and a great logical structure was built on this foundation. Apparently it was not widely recognized that the foundation could not support the structure. Both $\underline{P}$ and $\underline{B}^{0}$ contain the infinite-range couplings and other "fictitious" effects described above, so these equations cannot be accepted as a starting point for the theory.

Again the problem was first recognized by Bates and McCarroll [4] and they gave a partial resolution. A decade went by before an attempt at a more general theory was made by Mittleman [9], and it was another decade before an improvement upon his ideas was given $[1,10,19 \mathrm{a}]$. It was clear that somehow the effects of ETFs had to be incorporated, but since the ETF contains the classical nuclear velocity, it is intrinsically bound to the classical trajectory picture of atomic collisions, and it was not at all clear what the quantum-mechanical analog of an ETF might be.

The problem was resolved by considering the geometry of the potential surface and the associated wave function. Let us define mass-scaled coordinates

$$
\tilde{\mathbf{R}}=\mu^{1 / 2} \mathbf{R}, \quad \tilde{\mathbf{r}}=\mathrm{m}^{1 / 2} \mathbf{r} .
$$

In these coordinates, the kinetic energy operator becomes

$$
-\frac{\hbar^{2}}{2 \mu} \nabla_{R}^{2}-\frac{\hbar^{2}}{2 m} \nabla_{r}^{2}=-\frac{\hbar^{2}}{2}\left(\nabla_{\bar{R}}^{2}+\nabla_{F}^{2}\right) .
$$

It follows that the motion of electron and nuclei is equivalent to the motion of a single particle of mass $=1$ on a six-dimensional potential surface. A collinear cut of this potential surface is shown in Figure 2. A collisional wave function would correspond to a wave having an incoming part that propagates leftward up the $A$ channel toward the molecular region. Upon reaching this region, the wave is distorted in some complicated way, undergoing reflection, refraction, and diffraction in the double-well potential. Post-collision waves propagate back out of both channels. The amplitudes of outward-propagating waves in the $A$ channel are related to the probability of elastic scattering or of direct excitation of the electron to a higher $A$ atomic state, and the amplitudes of waves in the $B$ channel are related to the probability of charge exchange.

Let us now think about the form of the Ansatz (19) in this picture. The nodes of the product mainly arise from the nodes of $\chi(\mathbf{R})$, which are lines of constant R. Such nodes or wavefronts are shown in the top of Figure 3. Right away we can see that this picture is not a good one: nodal lines are supposed to be perpendicular to the direction of propagation of waves, but in this figure the nodal lines are not properly oriented relative to the direction of propagation down the channels.

We can get a better description by relating $\Psi$ to curved waves or to intersecting waves, as in the lower two parts of Figure 3. For example, if we define a curvilinear coordinate $\boldsymbol{\xi}(\mathbf{R} ; \mathbf{r})$ and expand $\xi$ as

$$
\Psi=\sum_{n} \chi_{n}(\xi) \phi_{n}(\mathbf{r} ; \xi)
$$




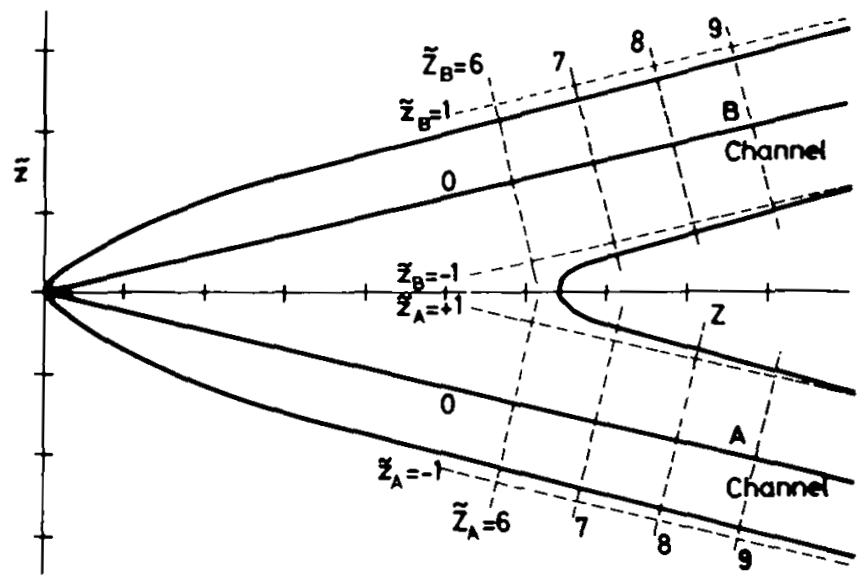

Figure 2. Colinear cut of potential surface in mass scaled coordinates. Electron and nuclei lie on a line, $\bar{Z}$ is the scaled distance between nuclei, and $z$ is the scaled distance from the center-of-mass of the nuclei to the electron. Heavy curves are equipotentials; the two straight lines have $V=-x$, where the position of the electron coincides with one or the other nucleus. Other curves have $V=-1$. Masses $M_{A}+M_{B}=9, m=1$ in this picture. For a real system, with $M_{A}, M_{B} \sim 2000$, the angle between the channels is narrower, but otherwise the picture is similar.
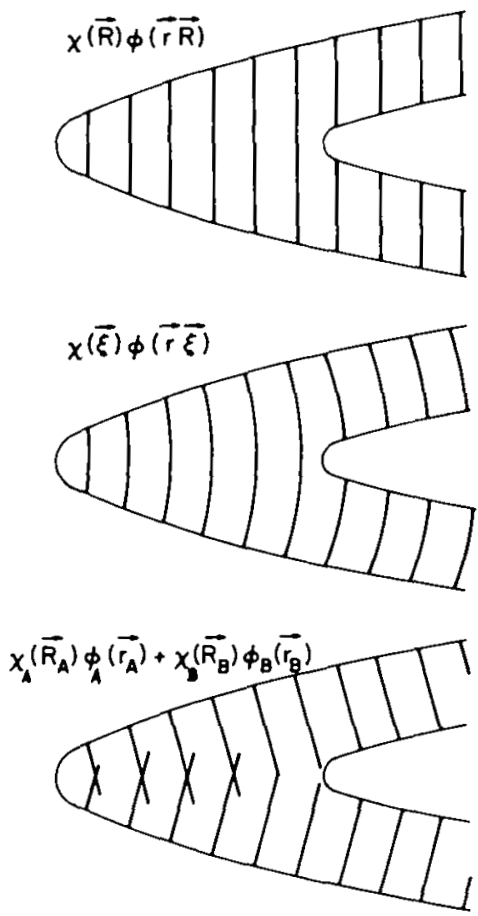

Figure 3. Nodal lines (or wave fronts) for various approximate wave functions. Simplest approximation (19) leads to the top picture, which is unsatisfactory because the wave fronts are not perpendicular to the direction of propagation. Curved waves or intersecting waves, as in the two lower pictures, provide a better representation. 
then the nodal lines of each product term in Eq. (25) may be curved in such a way that they are properly aligned with the channels. (A particularly simple special case is obtained if we use hyperspherical coordinates, and such an approach was used to develop an adiabatic theory of proton-transfer reactions [11].)

Alternatively, if the basis functions $\left\{\phi_{n}\right\}$ can be divided into $A$ - and $B$-centered states, we might define coordinates $\boldsymbol{\xi}_{A}, \boldsymbol{\xi}_{B}$ that are perpendicular to the channels. Intersecting wave trains, as in the lower part of Figure 3, would be given by

$$
\Psi=\sum_{n_{A}} \chi_{n_{A}}\left(\xi_{A}\right) \phi_{n_{A}}\left(\mathbf{r} ; \xi_{A}\right)+\sum_{n_{B}} \chi_{n_{B}}\left(\xi_{B}\right) \phi_{n_{B}}\left(\mathbf{r} ; \xi_{B}\right)
$$

A still more general approach would allow waves to be curved and to intersect; ie., for each basis function $\phi_{n}$ we choose a curvilinear coordinate $\xi_{n}$, and we expand

$$
\Psi=\sum_{n} \chi_{n}\left(\xi_{n}\right) \phi_{n}\left(r ; \xi_{n}\right)
$$

Starting from such an expansion, it can be shown that the functions $\chi_{n}\left(\xi_{n}\right)$ obey coupled integrodifferential equations, and that, by an expansion in powers of $(m / \mu)^{1 / 2}$, those integrodifferential equations can be reduced to differential equations. The analysis is long but the result is simple: the functions $\chi_{n}$ obey equations of the form

$$
\begin{aligned}
\left\{( 2 \mu ) ^ { - 1 } \left[\underline{\mathrm{S}}\left(-i \hbar \nabla_{R}\right)^{2}\right.\right. & +2(\underline{\mathbf{P}}+\underline{\mathbf{A}}+\boldsymbol{\gamma}) \cdot(-i \hbar \nabla)+\underline{\mathrm{B}}] \\
& +(\underline{\mathrm{h}+\mathrm{I}})-E\} \boldsymbol{\chi}=0
\end{aligned}
$$

These equations are similar in structure to Eqs. (21), but $\underline{P}$ has been replaced by $\underline{P}+\underline{A}+\underline{\gamma}, \underline{B}^{0}$ has been replaced by $\underline{B}$, and $\underline{h}$ by $\underline{h}+\underline{I}$. Definitions of all

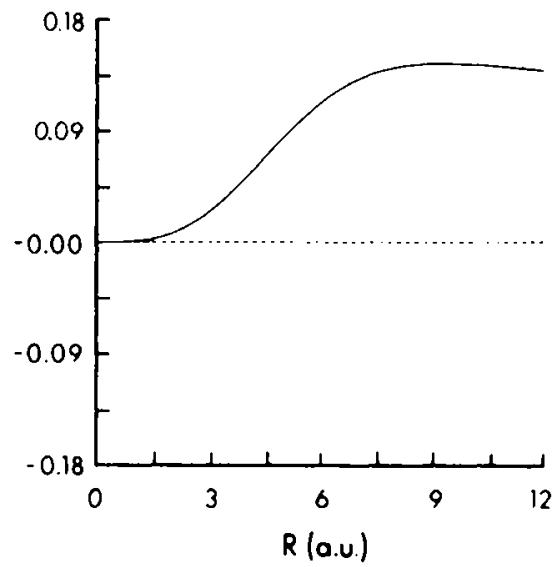

Figure 4. Radial coupling matrix element between the ground state of $\mathrm{HeH}^{2+}$ and a continuum state. $(-) P^{R}=\left\langle\phi_{\text {free }}|\partial / \partial R| \phi_{\text {ground }}\right\rangle(-\cdot)$ corresponding $P^{R}+A^{R}$, i.e., corrected nonadiabatic coupling when effects of displacement are removed using optimized switching functions. 
of these are given in Ref. 1. The essential point is that the difference between Eqs. (28) and (21) is directly analogous to the difference between Eqs. (16) and (6); i.e., the modifications to coupled equations that arise from ETFs in the classical trajectory framework should also be incorporated into the fully quantum-mechanical framework. They arise from the picture of intersecting curved waves.

Equations (28) have been postulated as a possible starting point for slow atomic collision theory. In Ref. 1 it was shown that most of the elaborately built-up structure of the theory can stand on this foundation with only minor modifications.

\section{Switching Functions}

The theory discussed above answers a number of long-unresolved questions about atomic collision theory, but it poses new ones. What is the "best" way of choosing switching functions $f_{n}(\mathbf{r} ; \mathbf{R})$ which specify the ETFs or the curvilinear coordinates? What choices will be "good enough" for a specific purpose? There is no easy way of answering these questions. Thorson and his co-workers have put a lot of effort into finding "optimized" switching functions for one-electron systems, and we briefly review some of their methods and results. (Other important optimization calculations are given in Ref. 19b.)

The first method they used involved minimization of nonspecific couplings. Thorson examined P-matrix elements for such ionization processes as

$$
\begin{aligned}
\mathrm{H}^{+}+\mathrm{H}(1 s) & \rightarrow 2 \mathrm{H}^{+}+e^{-}, \\
\mathrm{H}^{+}+\mathrm{He}^{+}(1 s) & \rightarrow \mathrm{H}^{+}+\mathrm{He}^{2+}+e^{-},
\end{aligned}
$$

and he found them to be unreasonably large and long range. He realized that this must be an artifact of the neglect of translation factors, and that these matrix elements really just represent the displacement of molecular states with the moving nuclei. By arduous calculations, it was found that for each molecular state one could find a switching function that would systematically reduce almost all of the coupling matrix elements, frequently by orders of magnitude. One illustration is given in Figure 4. Insofar as this method minimizes nonspecific coupling matrix elements, it should also minimize the error inherent in truncation of the expansions (27) or (15).

Later they derived switching functions by an analytical method. Since the electronic wave function is separable in prolate spheroidal coordinates $(\zeta, \eta, \phi)$, it can be expressed analytically by various types of series expansions. By close examination of the expansions for the "angle" dependent factor $S(\eta)$, Thorson et al. were able to decompose the wave function into two parts, each of which was associated with a single center. Generally the switching functions and coupling matrix elements obtained by this method agreed quite well with those obtained by the minimization method.

Both of the above methods rely upon the fact that the one-electron problem is exactly soluble. At present, we do not know how to make any sort of "optimal" 
choice of switching function for more complicated systems. One important result is available, however. Molecular electronic wave functions are usually obtained by expansions which ultimately rest upon single-center states:

$$
\phi_{\mu}(\mathbf{r} ; \mathbf{R})=\sum_{m} U_{m \mu}(R) \hat{\phi}_{m}(\mathbf{r} ; \mathbf{R}),
$$

where $\hat{\phi}_{m}$ is a state that is unambiguously associated with a definite center in the molecule (examples are Slater-type orbitals and Gaussian orbitals). Then the radial part of the $P$-matrix element is

$$
\begin{aligned}
\left\langle\phi_{\nu}\left|\frac{d}{d R}\right| \phi_{\mu}\right\rangle= & \sum_{m n} U_{n \nu}^{*}(R)\left\langle\hat{\phi}_{n} \mid \hat{\phi}_{m}\right\rangle \frac{d}{d R} U_{m \mu} \\
& +\sum_{m n} U_{n \nu}^{*}(R) U_{m \mu}\left\langle\hat{\phi}_{n}\left|\frac{d}{d R}\right| \hat{\phi}_{m}\right\rangle .
\end{aligned}
$$

In some early work, the first term was taken as an approximation, and the second term was neglected. At present it is more fashionable to include both terms, but several difficulties are noted. The second term depends upon the origin of coordinates, and, if it is properly calculated, it leads to some infinite-range couplings. However, it is not difficult to prove the following theorem: if the single-center states have no change with $R$ other than displacement with the moving nuclei (i.e., no variable orbital exponents, etc.), then the effect of the matrix $A^{R}$ is to cancel exactly the second term. It is therefore only the first term that is wanted. So long as the expansion is based upon single-center states which move with the nuclei but otherwise are fixed in character, the corrected radial nonadiabatic coupling matrix elements $\left(P^{R}+A^{R}\right)_{\nu \mu}$ arise only from changes in the coefficients $U_{m \mu}(R)$.

\section{A Cross Section}

To conclude, let us show a cross section for just one process for which experiments and calculations are available. In Figure 5 is shown the total exchange excitation cross section for

$$
\mathrm{H}^{+}+\mathrm{H}(1 s) \rightarrow \mathrm{H}(2 s)+\mathrm{H}^{+}
$$

There was an experiment by Bayfield [12] in which it was found that the cross section dropped quite steeply with decreasing proton energy below $10 \mathrm{keV}$. In 1976, Schinke and Krüger [13] calculated this cross section using an expansion like Eq. (5), i.e., ignoring ETFs, and including only angular couplings. Their results, while not in gross disagreement with Bayfield's data, do not represent a satisfying correspondence between theory and experiment. Subsequently, Crothers and Hughes [14] calculated this cross section by incorporating into the molecular expansion ETFs that are suitable for single-center states. Much better agreement with Bayfield's data was obtained. 


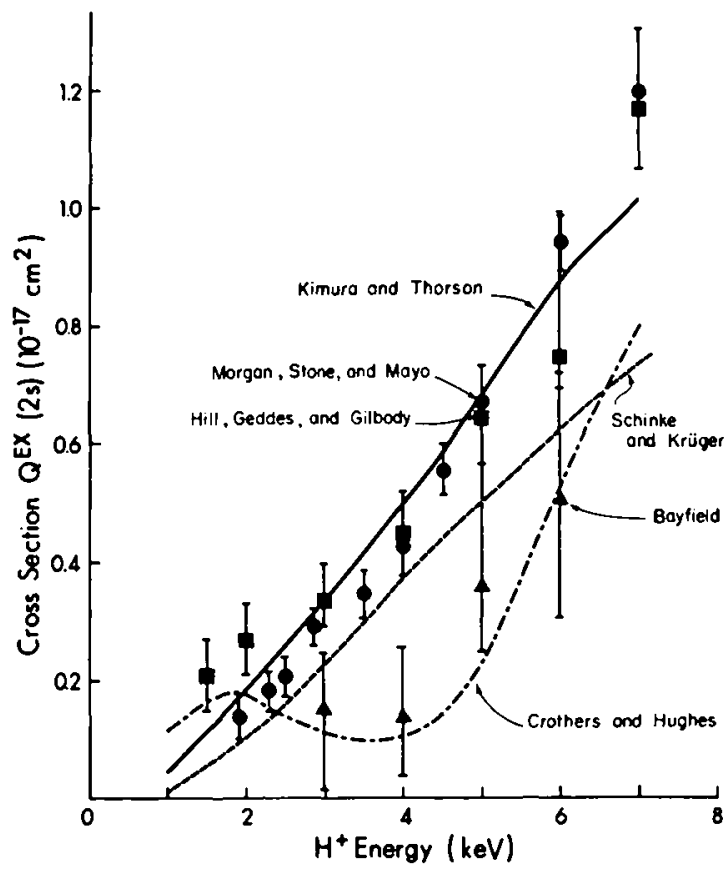

Figure 5. Cross section for $\mathrm{H}^{+}+\mathrm{H}(1 s) \rightarrow \mathrm{H}(2 s)+\mathrm{H}^{+}$

However, within the next couple of years there were two new measurements of the cross section by Morgan et al. [15] and by Hill et al. [16], and they found a significantly larger cross section. In 1981, the cross section was recalculated by Kimura and Thorson [17], using their analytically derived switching-function ETFs. This result is quite consistent with the new experiments [18].

\section{Acknowledgment}

This work was supported by the National Science Foundation.

\section{Bibliography}

[1] For further details, see J. B. Delos, Rev. Mod. Phys. 53, 287 (1981).

[2] N. F. Mott, Proc. Cambridge Philos. Soc. 27, 553 (1933).

[3] D. W. Jepsen and J. O. Hirshfelder, J. Chem. Phys. 32, 1323 (1960).

[4] D. R. Bates and R. McCarroll, Proc. R. Soc. London Ser. A 245, 175 (1958).

[5] R. McCarroll, Proc. R. Soc. London Ser. A 264, 547 (1961).

[6] S. B. Schneiderman and A. Russek, Phys. Rev. 181, 311 (1969).

[7] W. R. Thorson and H. Levy II, Phys. Rev. 181, 232 (1969); 181, 244, 252 (1969); C. F. Lebeda, W. R. Thorson, and H. Levy II, Phys. Rev. A 4, 900 (1971); V. SethuRaman, W. R. Thorson, and C. F. Lebeda, Phys. Rev. A 8, 1316 (1973); J. Rankin and W. R. Thorson, id. 18, 1990 (1979). 
[8] W. R. Thorson, M. Kimura, J. H. Choi, and S. K. Knudson, Phys. Rev. A 24, 1768 (1981); M. Kimura and W. R. Thorson, Phys. Rev. A 24, 3019 (1981).

[9] M. H. Mittleman, Phys. Rev. 188, 221 (1969); A 10, 442 (1974); M. H. Mittleman and H. Tai, Phys. Rev. A 8, 1880 (1973).

[10] W. R. Thorson and J. B. Delos, Phys. Rev. A 18, 117, 135 (1978); J. B. Delos, Phys. Rev. A 23, 2301 (1981).

[11] V. Babamov and R. A. Marcus, J. Chem. Phys. 74, 1790 (1981); V. K. Babamov, V. Lopez, and R. A. Marcus, J. Chem Phys. 78, 5621 (1983).

[12] J. E. Bayfield, Phys. Rev. 185, 105 (1969).

[13] R. Schinke and H. Krüger, J. Phys. B 9, 2469 (1976).

[14] D. S. F. Crothers and J. G. Hughes, Proc. R. Soc. London Ser. A 359, 345 (1978); Phil. Trans. R. Soc. 292, 56 (1979).

[15] T. J. Morgan, J. Stone, and R. Mayo, Phys. Rev. A 22, 1460 (1980).

[16] J. Hill, J. Geddes, and H. B. Gilbody, J. Phys. B 12, L341 (1979).

[17] M. Kimura and W. R. Thorson, Phys. Rev. A 24, 1780 (1981).

[18] It is not clear how much of the discrepancy between Refs. 14 and 17 results from the different translation factors that were used, and how much arises from other causes.

[19] (a) T. A. Green, Phys. Rev. A 23, 519, 532 (1981); (b) T. A. Green, E. J. Shipsey, and J. C. Browne, Phys. Rev. A 23, 546 (1981); 25, 1364 (1982); 27, 821 (1983).

Received May 4, 1983 